\title{
4. Konsiliardienst in der Notaufnahme - die hohe Kunst der zivilisierten Zusammenarbeit
}

\author{
Annegret Dreher und Ronald Burian
}

Grundlagen der Untersuchung und Behandlung von Patienten mit psychopathologischen Auffälligkeiten in der allgemeinen Notaufnahme (NA)

\section{Die Rollen des Psychiaters in der Notaufnahme}

Die konsiliarische Vorstellung eines psychisch auffälligen Patienten in der NA kann vor dem Hintergrund der unterschiedlichsten Erkrankungen erfolgen. So mag sich hinter einer Verhaltensauffälligkeit ein Delir, eine schizophreniforme Störung oder ein suizidales Syndrom verbergen. Vielleicht ist die Polizei vor Ort, eventuell ist der Patient psychomotorisch unruhig oder verbal aggressiv. Möglicherweise handelt es sich auch um einen Patienten, der sich einer lebensnotwendigen Untersuchung oder Behandlung verweigert, sich bedrohlich verhält, oder lautstark Suizidabsichten äußert. Derartig ,unbequeme“ Patienten generieren in der Regel ein hohes Maß psychischer Belastung und Anspannung unter dem gesamten medizinischen Personal einer NA. Der psychiatrische Konsiliararzt sieht sich nicht nur mit dem Patienten konfrontiert, sondern muss zusätzlich die Rolle eines Mittlers zwischen Patient und dem Team der NA einnehmen: Psychisch auffällige Patienten sind vom Betreten der NA an einem erhöhten Risiko der Fehlbehandlung ausgesetzt. 


\section{Häufige Störungen und Syndrome}

Akute Erregungs- und psychomotorische Unruhezustände sowie selbstschädigendes oder suizidales Verhalten machen einen Großteil psychiatrischer Notfälle aus. Die Erregungs- und Unruhezustände können sowohl Leitsymptom psychischer Erkrankungen als auch Ausdruck organischer Störungen einschließlich Intoxikationen sein. Alkohol ist sicher die häufigste Ursache für akute Intoxikationen, besonders gefährlich ist es aber, Mischintoxikationen, v.a. mit Alkohol und Medikamenten oder illegalen Drogen, zu übersehen.

Es ist in der Akutsituation sinnvoll, zunächst eine Syndrom-Diagnose zu stellen und die folgenden, vor allem somatisch-diagnostischen Schritte daraus abzuleiten. Besonders häufig in der Notaufnahme sind

- das verwirrt-delirante Syndrom (hyper- und hypoaktive Delirien - Ursache immer organisch),

- das paranoid-halluzinatorische Syndrom (Ursache kann organisch sein, oft aber Psychosen wie z.B. Schizophrenie),

- das katatone Syndrom/Erregungszustand (Ursache kann organisch sein, aber auch Psychosen oder dissoziative Zustände),

- das depressive Syndrom (schließt das suizidale Syndrom ein, organische Ursachen sind hier seltener, aber möglich),

- das ängstliche Syndrom (auch hier sind organische Ursachen und Entzugssyndrome möglich, häufiger aber Depressionen und Angststörungen).

Erneut: Die „organische Ursache“ schließt bei dieser Einteilung immer die Intoxikationen mit ein!

Die Einteilung nach Syndromen erscheint für die Notaufnahme effizient und handlungsleitend. Psychiatrische Differenzialdiagnosen sollten erwogen werden, eine ausführliche psychiatrische Abklärung bleibt oft der weiteren Behandlung vorbehalten.

Da das Übersehen von organischen Erkrankungen und Intoxikationen die häufigste Fehlerquelle für schlechte Verläufe und Komplikationen darstellt, ist eine genaue allgemein-körperliche und neurologische Untersuchung unerlässlich. Eine medikamentöse Behandlung dient in der Notaufnahme stets nur der Symptomkontrolle und sollte erst dann erfolgen, wenn andere situationsberuhigende oder vertrauensbildende Versuche fehlgeschlagen sind.

- In der Notaufnahme diagnostizieren wir pragmatisch in Syndromen und behandeln symptomorientiert.

- Körperliche Erkrankungen können durch psychische Auffälligkeiten verschleiert sein.

- Somatische Ursachen zu übersehen und eine körperliche Untersuchung zu unterlassen, nur weil sie schwierig ist, bedeutet jedoch eine Gefährdung für den Patienten. 


\section{Vorbereitung der Untersuchung}

Es ist eine Herausforderung, innerhalb limitierter Zeit und unter schwierigen Umgebungsbedingungen eine ruhige Gesprächsatmosphäre zu schaffen. Doch auch schon vor dem Gespräch mit dem Patienten sollten Sie folgende Dinge klären:

1. Bevor Sie den Patienten untersuchen, holen Sie sich Vorinformationen (Pflegepersonal, NA-Arzt, ggf. zuführende Polizei).

2. Wirkt der Patient agiert und/oder tritt er bedrohlich auf?

3. Gibt es Hinweise auf akute Suizidalität und/oder drohende bzw. erfolgte Selbstschädigung?

4. Spricht der Patient auf Deeskalationsversuche an; kann mit ihm die weitere Behandlung besprochen/verhandelt werden? Benötigt der Patient dringend eine Medikation?

5. Falls mehrere Akutpatienten gleichzeitig zu versorgen sind: In welchem Umfang benötigt der Patient Beaufsichtigung durch das medizinische Personal der Notaufnahme? Wird mehr Personal benötigt?

6. Befindet sich der Patient in einer ruhigen, sicheren Umgebung? Bestehen Sie ggf. auf ein ungestörtes Behandlungszimmer.

7. Nehmen Sie maximal einen Angehörigen oder einen professionellen Helfer mit dazu. Wenn Sie mit dem Patienten allein sind: Stellen Sie vorher sicher, wie Sie wen zu Hilfe rufen können, falls Sie in Gefahr kommen. Lassen Sie sich hierbei auf kein Risiko ein! Es gilt: Selbstschutz hat Vorrang!

Die Behandlung in der Notaufnahme beginnt damit, sich Vorabinformationen einzuholen, die helfen, von vornherein eine geeignete und sichere Untersuchungssituation zu schaffen.

\section{Systematisches Assessment}

Die zentralen Fragen, die Sie bei einer notfallmäßigen Erstvorstellung eines Patienten klären sollten, können im Rahmen eines klaren „Assessment-Plans“ schrittweise beantwortet werden:

Nutzen Sie die Checkliste Psychiatrisches Notfall-Assessment:

1. Welche psychischen Beschwerden und Erkrankungen führen den Patienten in die NA?

2. Welche körperlichen Beschwerden und Erkrankungen bestehen? Leidet der Patient an einer gravierenden körperlichen Erkrankung, die seine psychischen Beschwerden gänzlich oder zum Teil erklären könnte?

3. Gibt es entsprechende psychische und/oder körperliche Vorerkrankungen und Behandlungen? Nimmt oder nahm der Patient Medikamente ein? Spielen Alkohol oder Drogen eine Rolle? 
4. Erheben Sie einen psychiatrischen Befund im Rahmen des Möglichen. Klären Sie prädisponierende, auslösende und aufrechterhaltende Faktoren der zur Vorstellung führenden Beschwerden.

5. Versuchen Sie, fremdanamnestische Informationen von Menschen zu bekommen, die mit dem Patienten in der letzten Zeit Kontakt hatten (Partner, Verwandte, Hausarzt, Pflegedienst).

6. Erscheint der Patient in der Lage, für sich Entscheidungen zu treffen bzw. ist seine Einwilligungsfähigkeit maßgeblich beeinträchtigt?

7. Eigenhändige internistische und neurologische Untersuchung des Patienten. Sind weitere medizinische Untersuchungen nötig, um die zugrunde liegende Erkrankung zu identifizieren: Hier Rücksprache mit den ärztlichen Kollegen der anderen Disziplinen (Neurologie, Innere, Chirurgie?), ggf. Weiterleitung an andere medizinische Fachrichtungen.

8. Besprechen Sie auf dieser Basis mit dem Patienten die nächsten Untersuchungs- oder Behandlungsschritte und leiten Sie diese ein.

Frühzeitig sollten Sie den zeitlichen Verlauf bzw. die Entwicklung der Psychopathologie klären. Ist die Symptomatik akut aufgetreten oder hat sie sich über einen längeren Zeitraum entwickelt? Dies ergibt sich aus Ihrer Exploration und dem Zusammentragen sämtlicher über den Patienten verfügbarer Informationen; inklusive Labor- und apparativer Untersuchungsergebnisse und möglicher stationärer oder ambulanter Vorbehandlungen.

Ein einfach strukturierter „Assessment-Plan“ kann helfen, die Übersicht bei der Untersuchung zu bewahren. Einen solchen Plan kann man sich als Checkliste zurechtlegen und Punkt für Punkt abarbeiten.

\section{Professionelle Haltung und Übersicht}

Zielorientiertes Vorgehen und Besonnenheit sind im Notfall von besonderer Bedeutung, Die Ausstrahlung von Ruhe bzw. situationsberuhigende und vertrauensbildende Maßnahmen sind wichtig, gelingen aber gerade am Berufsanfang oft nur unter Anstrengung. Manchmal hilft es, einen tiefen ruhigen Atemzug zu nehmen, innerlich einen Schritt zurückzutreten und sich zu fragen:

- „Was ist jetzt am Wichtigsten und was kann erst mal warten?“ (first things first)

- „Was ist der nächste Schritt?“

Denken Sie daran, dass Sie immer nur eine Sache auf einmal machen können und konzentrieren Sie sich auf diese. Seien Sie fokussiert. Dabei hilft es, über den Tages- oder Dienstverlauf ein „Memo“ zu führen, in dem zu erledigende Aufgaben notiert sind. Das befreit von Gedächtnisaufgaben, die z.B. während eines Patientengespräches „im Hinterkopf ticken“. Seien nachsichtig mit sich selbst: Sie tun, was Sie aufgrund Ihres bisherigen Wissens tun können und bilden sich gewissenhaft fort. Lesen Sie nach, wenn Ihnen Wissenslücken auffallen. Aber stehen Sie dazu, wenn Sie einmal etwas nicht wissen und in einer Notfallsituati- 
on keine Zeit für aufwändige Recherchen bleibt. Kommunizieren Sie dies offen und holen Sie sich den erforderlichen Rat ein.

In der Notaufnahme kommt es ganz besonders darauf an, sich nicht in Details zu verlieren, sondern den Überblick über die wichtigsten Schritte und Aufgaben zu behalten. Wissenslücken müssen identifiziert und geschlossen werden, im Notfall jedoch scheuen Sie sich nicht, auf den Rat von erfahrenen Kollegen zurückzugreifen.

\section{Ansprache voreingenommener Patienten}

Nicht selten trifft man auch auf Patienten, die kein Verständnis dafür haben, von einem Psychiater untersucht zu werden. Einige haben das Bedürfnis, zu beweisen, dass sie „nicht verrückt“ sind. Diese Ängste oder Vorurteile verständnisvoll anzusprechen, ist essenziell, um überhaupt einen guten Erstkontakt zu ermöglichen. In diesem Zusammenhang ist vor allem die Ansprache wichtig: Von „psychiatrischem Vokabular“ wie „Depression“, „Panikstörung“ oder „Abhängigkeitserkrankung" werden solche Patienten eher abgeschreckt. Vielmehr sollte man zunächst in Umgangssprache beispielsweise von „Belastung“, „Stressreaktion“ bzw. „problematischem Trinken“ sprechen.

„Psycho-Slang“ ist nicht angebracht. Eine für den Patienten leicht verständliche Altagssprache erweist sich oft als Brücke zu einem guten Kontakt.

\section{Klärung der Einwilligungsfähigkeit}

Gerade wenn sich psychisch auffällige Patienten einer medizinischen Behandlung nicht unterziehen wollen (wenn beispielsweise der oben beschriebene Patient die empfohlene Lumbalpunktion ablehnt), ist dies für die Kollegen der somatischen Fachbereiche oft ein Grund, den Psychiater mit der Frage nach der Einwilligungsfähigkeit um Hilfe zu bitten.

Dass ein Patient psychisch auffällig ist, muss aber nicht bedeuten, dass er nicht in der Lage ist, Für und Wider einer angebotenen Behandlung gegeneinander abzuwägen. Ebenso wenig kann man aus dem anamnestischen Vorliegen einer psychischen Erkrankung (z.B. Schizophrenie) schließen, der Patient wäre per se nicht einwilligungsfähig.

Entscheidend ist, ob ein Patienten verstehen kann, aus welchem medizinischen Grund man ihm welche Behandlung angeboten hat und dies, auch $\mathrm{zu}$ einem späteren Zeitpunkt, in eigenen Worten wiederholen kann. Bei Ablehnung einer Behandlung durch den Patienten wäre zu klären, ob er die Risiken bei Unterlassung der Maßnahme verstanden hat. Um verstehen zu können, warum/worin er einwilligt bzw. ablehnt, muss er also über Art und Umfang einer medizinischen Behandlung in möglichst einfacher, verständlicher Sprache ins Bild gesetzt werden.

Grundsätzlich gilt: Die Frage nach der Einwilligungsfähigkeit („informed consent") eines Patienten bezieht sich stets auf eine konkrete Fragestellung bzw. die vorgeschlagene Behandlung. Die Einschätzung der Einwilligungsfähigkeit 
sollte nicht durch den Psychiater, sondern durch den aufklärenden somatischen Kollegen erfolgen, denn dieser ist der Experte für die jeweilig angebotene Untersuchungs- oder Behandlungsmaßnahme. Wenn der aufklärende Arzt berechtigte Zweifel an der Einwilligungsfähigkeit hat, kann der Psychiater hinzugezogen werden. In einem solchen Fall ist es empfehlenswert, den Patienten gemeinsam aufzuklären und zu befragen.

Das Einholen der Patienteneinwilligung ist die Pflicht des jeweils behandelnden Arztes. Als Psychiater begibt man sich bestenfalls in die Rolle eines Vermittlers im gemeinsamen Gespräch und verschafft sich so einen Eindruck, ob der Patient den aufklärenden Arzt versteht.

\section{Herausforderungen in der kollegialen und interdisziplinären Zusammenarbeit}

\section{Aufbau einer guten Zusammenarbeit}

Ein Einsatz in der NA kann Ihnen helfen, Ihre explorativen Fertigkeiten zu schulen, zu „verdichten“ und sich im „Krisenmanagement“ und in der Zusammenarbeit, aber auch in der Auseinandersetzung mit Kollegen anderer Fachrichtungen zu üben. Im interdisziplinären Diskurs ist Klarheit wichtig. In der Kooperation mit den ärztlichen Kollegen sollte klar festgelegt werden, wer für die Behandlung eines Patienten „hauptverantwortlich“ ist. Im Idealfall wird diese Entscheidung nicht telefonisch, sondern gemeinsam „am Patientenbett“ diskutiert und geklärt. „Knotenpunkte“ zwischen unterschiedlichen Fachrichtungen werden schnell zum unübersichtlichen „Knäuel“, in dem Behandlungspfade, die Gabe von Medikamenten oder ärztliche Anordnungen nicht mehr nachvollzogen werden können. Behandlungsfehler lassen sich besonders häufig auf schlichte Fehlkommunikation zurückführen.

Kollegialität beruht auf Gegenseitigkeit. Eine gute Strategie ist es, zu Beginn des Einsatzes in der NA grundsätzlich zu allen Kollegen, Ärzten wie Schwestern, freundlich, hilfsbereit und kollegial zu sein. Beobachten Sie gut, wie sich die Kollegen verhalten. Schon sehr bald werden Sie merken, welche Kollegen auch Ihnen gegenüber freundlich, hilfsbereit und kollegial sind, und welche abweisend und schmallippig werden, wenn sie Sie um Hilfe bitten. Diese Kollegen halten Hilfsbereitschaft oft für Schwäche, hier müssen Sie sich nicht ausbeuten lassen, sondern Klarheit und Konsequenz beweisen.

\section{Kommunikation mit unkollegialen Kollegen}

Eine Gesprächs-Sequenz mit einer fiktiven neurologischen Kollegin „Dr. L.“, die als neurologische Konsiliaria für die NA zuständig ist, soll die Grundsätze im Umgang mit unkollegialem Verhalten illustrieren:

Psychiaterin: „Frau Dr. L., ich möchte Sie bitten, sich den Patienten einmal neurologisch mit anzusehen, mir scheint, die akute Verwirrtheit kann Zeichen einer zerebralen Entzündung sein. Er klagt über Kopfschmerzen und hat eine Leukozytose im Blut."

Dr. L.: „Sie sind doch selbst Arzt, wenn Sie die Vermutung haben, machen Sie eine Lumbalpunktion.“ 
Hier können Sie jetzt bitten, argumentieren oder tatsächlich alles selbst machen, um einem Konflikt aus dem Weg zu gehen. Dies kostet jedoch wertvolle Zeit und birgt verschiedene Risiken bis hin zur Übernahme haftungsrechtlich relevanter Risiken. Wir schlagen Folgendes vor:

Psychiaterin: „Frau Dr. L., ich habe eine konkrete neurologische Verdachtsdiagnose. Ich muss jetzt hier in meinem Befund dokumentieren, dass Sie es ablehnen, bei dem Patienten ein neurologisches Konsil zu machen. Okay?"

Sollte Frau Dr. L. dann ihre Arbeit tun, ist es in Ordnung. Tut sie es nicht, dann dokumentieren Sie wie angekündigt die Weigerung und informieren Sie Ihren fachärztlichen Hintergrund. Dieser wird den Fall mit dem neurologischen Hintergrund besprechen, der die Sache dann wiederum mit Frau L. klären muss. Wenn sich solche Konflikte vor Ort nicht mit angemessenen Strategien kollegial klären lassen, ist der Wechsel der Hierarchie-Ebene notwendig und oft die wirksamste Intervention.

Sie selbst haben durch ihr eigenes Verhalten einen großen Einfluss auf die kollegiale Atmosphäre. Eine freundliche und kollegiale Zusammenarbeit ist erstrebenswert und fördert die Arbeitszufriedenheit. Gerade unter diesem Aspekt lohnt es sich aber auch, unkollegialen Kollegen offen entgegenzutreten.

\section{Fallbezogene Differenzen mit Kollegen anderer Fachrichtungen}

Als konsiliarpsychiatrisch tätiger Arzt in der NA werden Sie sich, wesentlich häufiger als im Rahmen der Tätigkeit in der Allgemeinpsychiatrie, mit älteren und auch geistig behinderten Patienten mit psychischen Auffälligkeiten konfrontiert sehen. Diese Patienten, deren psychische Auffälligkeiten häufig auf komplexen organischen Erkrankungen beruhen, sind, je nach Erregungs- oder Verwirrtheitszustand häufig „unbequem“ für somatische Abteilungen, da sie viel Zeit und Zuwendung verlangen. Trotz der soziodemographischen Entwicklung haben sich die meisten Krankenhäuser noch nicht ausreichend auf diese Patientenklientel eingestellt. Oftmals argumentieren Kollegen der somatischen Fachrichtungen dann, dass es z.B. doch „besser“ für einen älteren dementen Patienten sei, wenn er in der psychiatrischen Abteilung untergebracht würde, da diese doch besser auf die Behandlung von im Kontakt „schwierigen“ Patienten ausgelegt sei. Erneut: Gerade wenn es um den Ausschluss oder die Behandlung somatischer Ursachen einer psychischen Auffälligkeit geht, sollten Sie sich eher in der Rolle eines Fürsprechers bzw. eines „Patientenanwalts“ begreifen, der sich im Interesse des Patienten für die notwendige somatische Diagnostik einsetzt. Hier könnte eine Frage an den Kollegen der somatischen Disziplin lauten: „Meinen Sie wirklich, dass eine psychiatrische Abteilung besser auf die Behandlung einer Herzrhythmusstörung ausgelegt ist als eine internistische?“

Bei dementen und geistig behinderten Patienten kann die Rolle des Konsiliarpsychiaters manchmal die eines „Patientenanwalts“ sein, da diese Klientel durch Verhaltensauffälligkeiten in Gefahr gerät, bei körperlichen Erkrankungen ungenügend abgeklärt und behandelt zu werden. 


\section{Praktische Hinweise für die Gestaltung des Patientenkontaktes in der NA-Situation}

\section{Grundsätzliche Überlegungen}

Wie bereits oben angedeutet, besteht eine große Herausforderung darin, innerhalb kurzer Zeit unter „widrigen“ Umständen ein therapeutisches Verhältnis zum Patienten zu etablieren. Es gibt keine fest vorformulierte Vorgehensweise, um rasch ein therapeutisches Verhältnis zu einem Patienten aufzubauen, oder sich einen Eindruck von dessen Interaktionsverhalten und den Bewältigungsstrategien in Bezug auf eine vorliegende Erkrankung zu verschaffen. Doch möchten wir an dieser Stelle einige hilfreiche Strategien beschreiben.

Weiterhin kann es im Rahmen der Ersteinschätzung eines Patienten nützlich sein, sich im „eklektizistischen“ Sinne mit psychologischen Rahmenmodellen vertraut zu machen, die Anpassungsprobleme an psychische, soziale oder krankheitsbezogene Belastungen erklären können und damit einen Weg für den Psychiater aufzeigen, dem Patienten diese Anpassung zu erleichtern.

Im Folgenden sind zum einen pragmatische Ratschläge zur Optimierung der Explorationsatmosphäre aufgeführt. Zum anderen werden als Beispiel eines psychologischen Rahmenmodells unterschiedliche Typen erwachsenen Bindungsverhaltens kurz beschrieben. Letztere sollen als eine Art Raster dienen, um das Patientenverhalten besser zu verstehen und sich auf dieses besser einstellen zu können. Grundsätzlich hilfreiche Fragen dabei sind:

- Welches Beziehungsangebot braucht der Patient von Ihnen?

- Wie vermitteln Sie am besten Ihre therapeutischen Ratschläge, damit der Patient sie mit größtmöglicher Wahrscheinlichkeit umsetzt?

\section{Praktische Tipps zur Schaffung einer geeigneten Gesprächsatmosphäre}

Hinsetzen: Idealerweise haben Sie die Möglichkeit, die Anamneseerhebung dem Patienten gegenübersitzend durchzuführen, ggf. auch sitzend neben dem Kopf des Patientenbetts. So kann die Exploration auf „gleicher Augenhöhe“ stattfinden. Zudem wird dem Patienten vermittelt, dass Sie sich für die Exploration ausreichend Zeit nehmen und die Gesprächssituation nicht „fluchtartig“ wieder verlassen.

Etwas „Greifbares“ für den Patienten tun: Praktische, hilfreiche Gesten, wie das Reichen eines Wasserbechers oder das Adjustieren eines Kopfkissens können deeskalierenden Charakter haben. Sie vermitteln dem Patienten Vertrauen.

Die Hand reichen: Ein kurzer Händedruck zu Beginn und zum Ende einer Exploration können den Gesprächsbeginn und -abschluss markieren. Berührungen (in angemessenem Ausmaß) können in einigen Fällen die Distanz zwischen Ihnen und Ihrem Patienten reduzieren und ihm ein Gefühl von Sicherheit vermitteln. Insbesondere gilt dies für demenzerkrankte Patienten. Patienten, die mehr Distanz brauchen und den Händedruck nicht wünschen, vermitteln dies non-verbal. Es gilt, für diese Zeichen aufmerksam zu sein und die Bedürfnisse des Patienten zu akzeptieren. Keinesfalls sollte man die Distanzwünsche eines Patienten als 
persönliche Kränkung begreifen und selbst entsprechend ablehnend reagieren, auch und gerade wenn man ein eigenes Kränkungsgefühl bemerkt.

Den Patienten anlächeln: Dies gilt natürlich nur im geeigneten Kontext. Auch, oder gerade wenn Sie unter hohem Stress stehen, kann ein Lächeln ein durch den Patienten empfundenes Bedrohungs- oder Überforderungsgefühl deutlich entlasten.

Sich auf die „Sprache“ des Patienten einlassen: Dies bedeutet nicht, den Sprachduktus des Patienten gänzlich zu übernehmen, sondern in Worten zu sprechen, die dem Patienten verständlich sind. Dennoch kann es außerdem hilfreich sein, Formulierungen zu verwenden, die der Patient selbst vorgibt. Wenn der Patient beispielsweise im Rahmen eines depressiven Syndroms von „ständiger Erschöpfung“ spricht, ist es sinnvoll, dies aufzugreifen und beispielsweise einen Satz mit den Worten einzuleiten: „Trotz Ihrer ständigen Erschöpfung, ist es Ihnen da noch gelungen ...?“ Generell sollte ein vorsichtiger Umgang mit dem Wort „psychisch“ gepflegt werden (s. Abschn. „Ansprache voreingenommener Patienten“). Etwa bei einem psychoedukativen Gespräch mit einem Patienten mit einer somatoformen Störung sollten Formulierungen wie: „Das ist alles nur psychisch.“ vermieden werden.

Die Vorgeschichte mit eigenen Worten zusammenfassen: Sofern der Patient bereits von mehreren Ärzten in der Notaufnahme untersucht und exploriert wurde, kann es sich zeitsparend auswirken, die Umstände der Vorstellung bzw. die Vorgeschichte des Patienten als Gesprächseinleitung kurz mit eigenen Worten zusammenzufassen. Dies sollte dem Patienten allerdings die Möglichkeit einräumen, verfälschte Wahrnehmungen oder Fehleinschätzungen zu korrigieren und neue Aspekte der Vorgeschichte eingehender zu beleuchten.

Zudem bezeugt eine kurze Zusammenfassung der Umstände der Vorstellung die Tatsache, dass Sie sich bereits mit dem Fall des Patienten auseinandergesetzt haben bzw. im Vorfeld ein interdisziplinärer Austausch mit Ihren ärztlichen Kollegen erfolgt ist. Eine Zusammenfassung der Vorgeschichte durch einen Arzt, so rudimentär sie sein mag, kann auch für den Patienten wesentliche neue Aspekte eröffnen.

Was ist am vordringlichsten? Was macht den „Notfall“ aus? Worin besteht das unmittelbare Anliegen des Patienten? Sofern er gedanklich mit etwas anderem, einer möglichen Sorge oder Angst (beispielsweise „Wer versorgt zu Hause mein Kind.“) befasst ist, wird dies die Aufmerksamkeit von der Exploration ablenken. Manchmal ergibt sich nicht unmittelbar aus der Befragung eines psychisch erkrankten Patienten, warum (etwa statt einer Vorstellung beim ambulanten Psychiater) eine Vorstellung in der Notaufnahme erfolgen musste. Dies sollte dann klar erfragt werden. Möglichst frühzeitig das vordringlichste Anliegen des Patienten zu eruieren spart wertvolle Zeit und kann als „Leitfaden“ für den Explorationsverlauf dienen.

Was denkt der Patient über die eigene Erkrankung? Der Patient als „Sachverständiger“ der eigenen Störung: Welche sind die größten mit der Symptomatik verbundenen Ängste? Diese Frage bietet eine weitere Möglichkeit, Fehlannahmen über die Erkrankung frühzeitig zu korrigieren. Eine zeitnahe Klärung kann sich positiv auf die Com- 
pliance des Patienten im weiteren Behandlungsverlauf auswirken. Welche Mutmaßungen hat der Patient selbst über seine Erkrankung angestellt? Hat er Vermutungen bezüglich Belastungs- oder pathogenetischer Faktoren, die seine Erkrankung oder Störung bedingt haben könnten? Diese Explorationsstrategie kann wesentliche diagnostische Hinweise liefern.

Den Patienten zum „Verbündeten“ im Rahmen der weiteren Diagnostik machen, ggf. Parallelen zur somatischen Medizin herstellen: Gerade die Durchführung kognitiver Testungen, etwa des MMST kann im Rahmen einer „notfallmäßigen“ Exploration auf den Patienten sehr abrupt wirken und eher zum Aufbau von Misstrauen und Feindseligkeit führen. In jedem Fall sollten Sie erklären, warum Sie beispielsweise eine kognitive Testung durchführen.

Abhilfe kann die folgende Vorgehensweise schaffen: „Bitte unterstützen Sie mich dabei, herauszufinden, was die Konzentrationsschwierigkeiten bei Ihnen verursacht. Dazu würde ich Ihnen gerne einige Fragen stellen. Diese Fragen sind für mich ein wichtiges Untersuchungsinstrument und werden vielleicht bei Ihrer Weiterbehandlung wiederholt werden müssen, ähnlich wie Sie das von Untersuchungen Ihres Blutbilds kennen.“ Eine „ko-investigative“ Rolle befreit den Patienten zudem aus der des „passiven Krankheitsopfers“.

Die Notlage des Patienten anerkennen: Für Patienten kann es sehr beruhigend sein, sich mit den eigenen Befürchtungen und dem eigenen Leidensdruck angenommen, „erkannt“ zu fühlen. Gerade bei Patienten mit somatoformen Störungen kann eine Validierung bzw. Anerkennung der empfundenen Beschwerden - entgegen der unmittelbaren „Enttarnung“ der Psychogenese einer Erkrankung - ein wichtiger therapeutischer Schritt sein.

Dem Patienten „Feedback“ geben: In jedem Fall sollte dem Patienten orientierend die Verdachtsdiagnose, das weitere Untersuchungs- und Behandlungskonzept erläutert werden, auch wenn nicht jeder Schritt im Detail auseinandergesetzt werden muss: Der Patient sollte eine ungefähre Vorstellung von dem haben, was ihn erwartet. Stellen Sie Verständnisfragen gegen Ende des Arztgesprächs, z.B.: „Nun habe ich so viel geredet. Jetzt würde ich gern hören, ob es bei Ihnen auch richtig angekommen ist. Können Sie mir kurz sagen, wie Sie es verstanden haben: Welche Art von Erkrankung vermuten wir bei Ihnen und welche nächsten Untersuchungsschritte planen wir?"

\section{Bindungstheoretischer Exkurs: Eine zusätzliche „Brille“ zur Beurteilung interaktio- nellen Verhaltens}

Die Kenntnis von bindungstheoretischen Konzepten bietet eine Möglichkeit, Behandlungsstrategien besser an die Interaktionsmuster und Bedürfnisse der Patienten anzupassen. Zudem stellen diese Konzepte ein zusätzliches Werkzeug dar, auch nicht-psychiatrischen Kollegen das Verhalten „schwieriger“ Patienten besser verständlich zu machen. Die Klassifizierung bzw. das Kennenlernen der unterschiedlichen Bindungstypen kann für den Arzt in der Notaufnahme eine Art diagnostischer „Brille“ darstellen, die „schnell aufzusetzen“ ist und mit deren Hilfe die Kontaktaufnahme und Gestaltung der Arzt-Patienten-Beziehung we- 
sentlich erleichtert wird. Ein wichtiges Instrument der Bindungsstil-Diagnose ist nicht nur die genaue Verhaltensbeobachtung des Patienten, sondern die Beobachtung der eigenen Empfindungen, also möglicherweise der Impulse von Fürsorge und Nähe, aber auch von Gefühlen wie Misstrauen, Hilflosigkeit oder Wut, die ein Patient im untersuchenden Arzt auslöst.

Die Bindungstheorie umschreibt evolutionäre und entwicklungsgeschichtliche Wurzeln der Muster zwischenmenschlicher Beziehungen. Diese Muster resultieren aus einem Zusammenspiel von Umwelt- (hier v.a. die Beziehung zu den Eltern) und genetischen Faktoren und persistieren relativ stabil über den Verlauf des Lebens. Die Entwicklung eines bestimmten Bindungsstils bei einem Kind, die zunächst aus der Interaktion mit ihrem primären Versorger resultiert, hat die eigentliche Funktion, den erlebten Stress für das Kind - beispielsweise bei Trennung von der Bezugsperson - so minimal wie möglich zu halten. Diese Anpassungsprozesse an ihre primären Bezugspersonen erlauben Kindern im späteren Verlauf zügige Rückschlüsse auf andere zwischenmenschliche Interaktionen. Die Entwicklung eines spezifischen Bindungsstils bei einem Kind soll also im Grunde die Effektivität sozialer Interaktion erhöhen und es vor Belastung schützen.

Die Spuren dieser Interaktionsstile setzen sich bis ins Erwachsenenalter fort und können bei Patienten nicht nur einen deutlichen Einfluss auf die Interaktion mit dem Arzt haben, sondern auch auf Symptomverstärkung, Gesundheitsverhalten und das Einholen sozialer Unterstützung. Dies ist vor allem durch die Tatsache bedingt, dass die Bindungsmuster durch Situationen reaktiviert werden, die einen starken psychischen Stressor darstellen: Beispielsweise eine Vorstellung im Krankenhaus, eventuell sogar in der Notaufnahme, beeinträchtigte kognitive Fähigkeiten oder auch die Trennung vom gewohnten sozialen Umfeld. Weiterhin werden diese Bindungsprozesse durch Situationen ausgelöst, in denen es notwendig ist, Vertrauen $\mathrm{zu}$ einem Interaktionspartner - z.B. dem anamneseerhebenden Arzt - aufzubauen. Auch hier dient die Reaktivierung „erlernter“ Bindungsmuster der Regulation von Stress.

Im Folgenden sollen vier „Prototypen“ erwachsenen Bindungsverhaltens umschrieben werden, wie sie von Bartholomew und Horowitz (1991) herausgearbeitet wurden; vor allem in Bezug auf ihre Implikation für den ärztlichen Erstkontakt zu einem Patienten in der Notaufnahme. In jedem Fall ersetzen die folgenden Beschreibungen keinesfalls eine psychiatrische Diagnostik, hier geht es jedoch um Persönlichkeitsstile und -akzentuierungen. Zudem gibt es natürlich Patienten, die Charakteristika mehrerer der unten angeführten „Prototypen“ aufweisen. Diese Klassifizierung stellt allerdings den Versuch einer Simplifizierung des zugrundeliegenden „Kontinuums“ dar, auf dem sich das menschliche Interaktionsverhalten bewegt.

Der sichere Bindungsstil: Patienten mit sicherem Bindungsverhalten haben ein suffizientes Selbstvertrauen und Vertrauen in die Notwendigkeit bzw. den Wert enger zwischenmenschlicher Beziehungen. In ihrer Entwicklung durften sie ein weitgehend konsistentes, „bedarfsgesteuert“ vermitteltes Gefühl von Sicherheit durch ihre Eltern bzw. ihre Primärversorger erleben. Sie können flexibel in Rollen zwischen Autonomie und Abhängigkeit changieren und sich von allen Bindungs- 
typen am besten an widrige Umstände anpassen. Für den medizinischen Kontext bedeutet dies, dass die „Stress-Antwort“ des Patienten auf eine Erkrankung am ehesten proportional zu den bedingenden Auslösern ausfällt und häufig für den behandelnden Arzt am ehesten nachvollziehbar ist. In Untersuchungen unter Angehörigen von Gesundheitsberufen konnte nachgewiesen werden, dass diese gegenüber Patienten mit sicherem Bindungsstil am ehesten Empathie empfinden und es leicht erscheint, die Anamnese eines solchen Patienten zu verstehen, Behandlungsratschläge zu kommunizieren und konstruktives, problemlösendes Verhalten bei diesen Patienten zu mobilisieren.

Der besitzergreifende Bindungsstil (im Englischen: „preoccupied“): Dieser Bindungsstil ist durch „abhängiges“ Verhalten, einem verstärkten Ausdruck von Emotionen und einem intensiven Bedürfnis nach der Aufrechterhaltung von emotionaler Nähe charakterisiert. In ihrer Entwicklung erfuhren diese Patienten eine inkonsistente Vermittlung von Sicherheit und Fürsorge. Ihre Interaktionsmuster können als Resultat eines „hyperaktivierten“ Bindungssystems verstanden werden: Ein chronisches Gefühl von Verletzbarkeit oder Gefahr löst eine defensive, überschießende „Stress-Antwort“ aus. Der Patient ist fortwährend damit beschäftigt, emotionale, gar physische Nähe zum Bindungspartner aufrechtzuerhalten. Im medizinischen Setting zeichnen sich diese Patienten durch ein hohes Maß an Hypervigilanz aus, die ihre Aufmerksamkeit - beispielsweise für körperliche Symptome deutlich verstärken kann. Patienten sind im Rahmen der Exploration eher mit sich und ihren eigenen Emotionen beschäftigt. Für den Arzt kann es sich schwierig gestalten, „harte Fakten“ aus der Krankengeschichte herauszuarbeiten. Ständige Beschäftigung mit der Sorge um eine mögliche oder die bestehende Erkrankung kann hier zur übermäßigen Inanspruchnahme des medizinischen Versorgungssystems führen, etwa im Sinne von „Doctor-Hopping“ oder häufigen Notfallvorstellungen. Diesen Patienten begegnet das medizinische Personal häufiger mit Abstand oder Vermeidung, was aber das hilfesuchende und anklammernde Verhalten paradox verstärkt.

Der abweisende Bindungsstil (im Englischen: „dismissing“): Dieses Bindungsverhalten wird durch ein hohes Maß an Autarkie und einem größeren Maß zwischenmenschlicher Distanz und Zurückhaltung bestimmt. In ihrer Entwicklung wurden diese Patienten von ihren Primärversorgern überwiegend teilnahmslos behandelt bzw. erfuhren diese als wenig umsorgend und schützend. Typischerweise gehen Patienten mit diesem Bindungsstil eher misstrauisch in ungewohnte, stressauslösende Interaktionen. Situationen, die ein gewisses Maß an Intimität oder Abhängigkeit abverlangen, wirken auf solche Patienten eher aversiv. Nähe zum „Bindungspartner“, wie z.B. einem NA-Arzt, wird daher eher vermieden und Signale persönlicher Schwäche werden unterdrückt. Ein positives Selbstwertgefühl wird dadurch reguliert, dass Andere auf Distanz gehalten oder abgewertet werden. Es besteht wenig Zugang zu den eigenen Emotionen. Symptome werden von diesen Patienten ggf. erst spät berichtet oder heruntergespielt. Die Entschlossenheit, Probleme „auf die eigene Art“ zu lösen, könnte zu ComplianceSchwierigkeiten führen und tatsächlich empfundener Stress eher „maskiert“ werden, was bei diesen Patienten zu längerfristigen Problemen führen könnte. 
Der unsichere Bindungsstil: Bei diesem Bindungstypus wird ein inkonsistentes Spannungsfeld zwischen erhöhter Bindungsvermeidung und dem Bedürfnis nach Nähe zum „Bindungspartner“ deutlich. Den kindlichen Bedürfnissen wurde hier mit intensiver Abneigung bzw. mit Härte durch ihre primären Versorgungspersonen begegnet. Vertreter dieses Bindungsstils sind häufiger im medizinischen Setting präsent als Patienten mit abweisendem Bindungsverhalten. Beide Stile unterscheiden sich deutlich im verbal oder über das Verhalten kommunizierten Maß an Stress, wobei letzterer für den unsicheren Bindungsstil überschießende Ausmaße annehmen kann. Ein Mensch mit unsicherem Bindungsverhalten ist anfällig für mögliche soziale Isolation, aber wirkt im Gegensatz zum oben beschriebenen Typus nicht selbstbewusst und selbständig. Mit den Vertretern des „besitzergreifenden“ Bindungsstils teilen sie ein niedriges Selbstbewusstsein und eher negativen Affekt. Die Nutzung des Gesundheitssystems resultiert bei diesen Patienten häufig in einer schwierigen, ambivalenten Haltung zwischen der Signalisierung eines bestehenden Leidensdrucks und der Ablehnung medizinischer Behandlung im Sinne von: „Ich leide, hilf mir, aber komm mir nicht zu nahe.“

\begin{abstract}
Ein Fallbeispiel
Zur Verdeutlichung der Praxisrelevanz einer Beachtung des Bindungsstils stellen wir hier eine fiktive Gesprächssequenz mit einem Patienten in der NA vor. Herrn A. ist ein Automechaniker, der sich wegen akuter Brustschmerzen in der NA vorstellte. Die internistischen Kollegen hatten zunächst einen Myokardinfarkt ausgeschlossen, stellten Herrn A. dann aber wegen Schlafstörungen, Appetitlosigkeit und deutlich gedrückter Stimmung nach partnerschaftlicher Trennung mit dem Verdacht eines depressiven Syndroms dem Psychiater vor.
\end{abstract}

Psychiater: „Guten Tag Herr A., mein Name ist D. und ich arbeite als Psychiater in der Notaufnahme. Mein Kollege Herr B. hat mich gebeten, mit Ihnen zu sprechen und einige Fragen zu Ihren Beschwerden zu stellen ..."

Herr A. (unterbricht den Psychiater): „Oh Gott, jetzt kommt auch noch der Psycho-Klempner! Das hatte ich befürchtet! Ich bin nur wegen meiner Brustschmerzen hier. Und für meine Schlafstörungen wollte ich lediglich ein Mittelchen zum Einschlafen!“

An dieser Stelle könnte der Psychiater dem Patienten ein ähnlich aversives Verhalten entgegenbringen, wie dieser ihm gegenüber aufweist und gereizt reagieren. Allerdings reflektiert er, dass es bei dem Patienten Hinweise auf ein depressives Syndrom gibt und zudem möglicherweise ein „aktiviertes Bindungssystem" hinter seinem ablehnenden Verhalten steht. Offenbar reagiert der Patient mit Wut und zwischenmenschlicher Distanzierung auf eine unbekannte Situation, was für einen abweisenden Bindungsstil sprechen könnte. Nun geht es darum, einen Zugang zu dem Patienten zu gewinnen, indem der Psychiater sich dem vermuteten Distanzbedürfnis des Patienten anpasst:

Psychiater: „Herr A., ich möchte Ihnen gar keine Behandlung aufzwingen, schon gar keine psychiatrische. Kann ich Ihnen vielleicht ein Angebot machen?"

Der Psychiater macht klar, dass er die Grenze des Patienten respektiert und überlässt Herrn A. die Wahl.

Herr A.: „Nicht gern. Aber lassen Sie hören.“

Psychiater: „Wir können im Rahmen einer kurzen Untersuchung versuchen, herauszufinden, was die Ursache Ihrer Schlafstörungen sein könnte. Dazu muss ich Ihnen vor allem ein paar Fragen stellen, die mir helfen, Ihre Schlafstörungen genauer einordnen zu können, okay?“

Herr A.: „la gut, und dann?“

Psychiater: „Wenn wir zu dem Ergebnis kommen, dass eine bestimmte Erkrankung die Ursache ist, steht es Ihnen immer noch frei, zu entscheiden, ob Sie meine Behandlungsvorschläge dann annehmen oder nicht. Ich habe keine Ahnung von Autos, aber ich stelle mir das so vor: Wenn ich Ihnen einfach etwas zum Einschlafen verschreiben würde, ohne dass ich die Ursache weiß, wäre das, als hätte mein 
Auto ein Öl-Leck und ich immer wieder Öl nachgießen würde, ohne zu schauen, wo das Auto Leck geschlagen hat."

Herr A. (grinst): „Na gut, schießen Sie los.“

Der Psychiater hat in dieser Gesprächssequenz dem Patienten stets Wahlmöglichkeiten angeboten, hat Worte wie „Angebot“ und „freie Entscheidung“ benutzt. Zudem konnte er inn in seiner beruflichen Expertise wertschätzen, indem er den Auto-Vergleich anstellte und gleichzeitig andeutet, dass er, der Psychiater (im Gegensatz zu Herrn A.) auf diesem Gebiet „keine Ahnung“ habe. Dies half offenbar, das Gespräch gut anzubahnen.

\section{Hilfreiche Strategien in der Beziehungsgestaltung}

Zusammenfassend lassen sich die Regeln der ärztlichen Beziehungsgestaltung bei den vier exemplarischen und zu didaktischen Zwecken stark vereinfachten Bindungsstilen wie folgt beschreiben:

1. Bei Patienten mit sicherem Bindungsstil müssen in der Regel im ärztlichen Kontakt keine Besonderheiten beachtet werden.

2. Patienten mit besitzergreifendem Bindungsstil benötigen einen „führenden“, klar strukturierten Stil, aber viel Unterstützung bei der Entscheidungsfindung.

3. Ein distanzierter Bindungsstil erfordert es, dem Patienten zwar alle Informationen an die Hand zu geben, aber ihm ein Höchstmaß an Entscheidungsfreiheit im therapeutischen Prozess zu überlassen.

4. Patienten mit unsicherem Bindungsstil stellen durch ihr Interaktionsverhalten oft besonders große Herausforderungen an das therapeutische Geschick. Hier kommt es darauf an, Sicherheit zu vermitteln, aber gleichzeitig die persönlichen Grenzen des Patienten zu achten.

\section{Literaturempfehlung}

Bartholomew K, Horowitz L (1991) Attachment Styles Amoung Young Adults: A Test of a Four-Category Model. Journal of Personality and Social Psychology 61, 226-244 